\title{
Procedural Sedation of Preterm Neonate with Frequent Apnea during Magnetic Resonance Imaging: A Case Report
}

\author{
Jaewoong Jung, Juhui Park, Yang Hoon Chung, Won Seok Chae \\ Department of Anesthesiology and Pain Medicine, Soonchunhyang University Bucheon Hospital, Soonchunhyang University College of Medicine, Bucheon, \\ Korea
}

\begin{abstract}
Magnetic resonance imaging (MRI) is a useful and safe imaging modality for examining preterm infants. However, MRl examination requires careful precautions, and infants and children are likely to require deep sedation or anesthesia to keep them still during the examination. Sedation has various risks and the greatest concern of sedation is cardiorespiratory events. In addition, delicate titration is also necessary for preterm infants because propofol pharmacokinetics is different from those in older children. We successfully completed sedation of a preterm neonate (gestational age, 32+1 weeks; birth weight, 1,970 g) with a history of frequent apnea through careful assessment and continuous monitoring. We want to suggest alternative options for airway management of the high risk of respiratory complications.
\end{abstract}

Keywords: Premature infant; Sleep apnea syndromes; Deep sedation; Case report

\section{INTRODUCTION}

Preterm birth is considered risky because immaturity itself is accompanied by mild to severe diseases or congenital anomalies. Therefore, when a preterm infant is born, careful physical examination and imaging modalities such as ultrasonography, computed tomography, and magnetic resonance imaging (MRI) are needed to find comorbidity and provide proper treatments.

Especially for preterm infants, MRI is useful in examining detailed images of the developing brain and congenital malformations and detailed patterns of brain injury that are essential for prognostic plans [1]. Although MRI is considered relatively safe because it does not use radiation, it requires careful precautions due to hazardous magnetic field interactions [2]. The unique environment disturbs the immediate approach to a patient's airway during the event of desaturation and restricts the use of usual metallic or electric airway devices such as videoscope. MRI-compatible anesthetic equipment is essential. Moreover, infants and children are more likely to require deep sedation or anesthesia to resolve anxiety and keep them still during examination in a noisy and cylindrical closed space [3].

We report our sedation case of a preterm neonate with a history of frequent apnea. We want to suggest alternative options for airway management of the high risk of apnea during procedural sedation for an MRI scan.

\section{CASE REPORT}

The patient was born at $32+1$ weeks $(1,970 \mathrm{~g})$ by caesarean section due to maternal preterm premature rupture of membranes. The gender expressed by external genitalia was female. Apgar scores at 1-, 5-, and 7-minute were 4, 6, and 9, respectively. She was transported to the neonatal intensive care unit (NICU). Her limb activity was poor and chest retraction was found. The Pediatrician decided to perform intubation and began to inject caffeine citrate. The next day, her respiration rate became regular. Her chest retraction was not observed anymore. The endotracheal tube was removed and the high-flow nasal cannula was applied. Peripheral capillary oxygen saturation $\left(\mathrm{SpO}_{2}\right)$ was well maintained over $97 \%$. However, bradycardia with brief apnea episodes occurred fre- 
quently. After 5 more days of caffeine citrate treatment, the frequency of bradycardia with brief apnea gradually decreased. On physical examination, masses were detected in both inguinal areas. Abdominal ultrasonography revealed no visible uterus with soft tissue attenuations at both inguinal canals. For evaluating the condition of structures in her pelvic cavity, a pelvic MRI was necessary. At the same time, the result of cranial ultrasonography suggested suspicious brain injury. Therefore, brain MRI was also required for accurate diagnosis and prognosis.

For the MRI scan, the pediatrician prescribed her chloral hydrate syrup. However, the MRI scan failed 4 times due to inadequate sedation, movement, and the event of desaturation. After that, transient desaturation was detected during feeding. The pediatrician consulted us about procedural sedation during an MRI scan. At that time, her corrected gestational age was $36+5$ weeks and her body weight was 2,670 g.

First of all, we assessed her medical history and physical status, mainly focusing on the airway. There was no morphological evidence indicating difficult airways such as the short neck, limited mouth opening, or small mandible. Although the episodes of bradycardia with brief apnea were alleviated, the episode of transient desaturation during feeding made us worry about airway safety during sedation. For those reasons, we discussed with the pediatrician and planned the following: intubation at NICU, transportation to MRI suite under sedation, and MRI scan with continuous monitoring. The reason why we performed intubation at NICU was that the MRI suite was an unfamiliar environment. In addition, it was far from the operating room. In case of emergency, it would be hard to call for another anesthesiologist's help. At the NICU, the preterm neonate was monitored and induced sedation with the loading of propofol at $1 \mathrm{mg} / \mathrm{kg}$ after preoxygenation. Intubation was performed without using a muscle relaxant. Careful auscultation and end-tidal carbon dioxide $\left(\mathrm{EtCO}_{2}\right)$ monitoring through capnography suggested successful intubation. We started to infuse propofol at a rate of $100 \mathrm{mcg} / \mathrm{kg} / \mathrm{min}$. After regular selfrespiration and maintenance of $\mathrm{SpO}_{2}$ were guaranteed, we transported her to the MRI suite cautiously with continuous monitoring of $\mathrm{SpO}_{2}$, electrocardiogram (EKG), non-invasive blood pressure (NIBP), and $\mathrm{EtCO}_{2}$ while supplying oxygen. At the MRI suite, we changed all monitoring equipment and infusion pumps to those that were MRI-compatible. The $\mathrm{SpO}_{2}, \mathrm{EKG}, \mathrm{NIBP}$, and $\mathrm{EtCO}_{2}$ monitoring were maintained continuously during the MRI scan. Additional sedatives were not necessary as well as changes in the dosage of propofol. The MRI scan was performed successfully with immobilization. Patent airway had regular self-respiration, and oxygen was supplied at a rate of $5 \mathrm{~L} / \mathrm{min}$ during the entire procedure. The total duration of the MRI scan was 91 minutes. Since there was no MRI compatible ventilator at that time, so medical staff including anesthesiologists and pediatricians were on standby with equipment such as Ambu-bag and drugs such as atropine and epinephrine. Fortunately, there was no adverse event such as desaturation, apnea, or bradycardia. At the end of the MRI scan, propofol infusion was stopped and she was transported to NICU with continuous monitoring. The endotracheal tube was removed within 30 minutes after arriving at NICU. There were no other complications during her hospital convalescence.

This study was approved by the institutional review board of Soonchunhyang University (IRB approval no., 2019-10-006) and the requirement for informed consent was waived because all data were obtained by retrospective chart review.

\section{DISCUSSION}

Preterm infants are known to have more apnea events postoperatively. Further, the sedation of preterm infants with frequent apnea for MRI scans is somewhat different from other procedural sedations. During the MRI scan, the patient was left in a cylindrically closed space of the MRI machine and the medical staff could not approach the patient easily. The medical staff could only observe the patient through the camera and identify the patient's status through a monitoring screen. Even if an adverse event occurs, it is hard to take immediate action. For these reasons, we concluded that tracheal intubation was necessary for the safety of this preterm neonate.

Many guidelines recommend us to follow the following principles to avoid adverse events: pre-sedation assessment, informed consent, appropriately prepared equipment, proper selection and dose of sedative agents, monitoring during the procedure, and discharge criteria [2]. Reviews of patients' medical histories and physical examinations are important factors to determine who will provide the sedation, what sedative drug should be used, and which level of sedation will be targeted. Especially, airway examination and medical conditions that may affect a patient's response to sedating medication are the key factors of pre-sedation assessment. When an increased risk of sedation is expected because of a patient's underlying medical conditions, almost all guidelines rec- 
ommend consultation with appropriate subspecialists or anesthesiologists who are skilled in advanced airway management [4]. Special care by the physician or anesthesiologist who is trained in advanced airway management is needed.

Propofol is considered an ideal sedating agent for non-painful procedures such as MRI scans or nuclear scans [2]. The advantages of propofol are its short induction time, more rapid recovery, and lower failure rate $[5,6]$. To titrate propofol for sedation, a standardized protocol is needed by the anesthesiologist or sedationtrained pediatrician (bolus dose of 1 to $2 \mathrm{mg} / \mathrm{kg}$, infusion at a rate of 100 to $200 \mathrm{mcg} / \mathrm{kg} / \mathrm{min}$ ) [6]. Throughout all procedures, the maintenance of spontaneous ventilation must be verified by continuous monitoring $\mathrm{EtCO}_{2}$ with capnography because propofol can make the level of sedation become too deep and unexpectedly induce respiratory depression and hypoxemia [2,7]. Guidelines published by specialists at their own practices recommend the administration of propofol conducted by the medical staff qualified for advanced airway management and resuscitation in an emergency [8].

Propofol pharmacokinetics in preterm infants is different from that in older children, especially in terms of clearance. Preterm infants are more likely to show reduced clearance. They have an increased risk for accumulation during intermittent bolus or continuous infusion of propofol [9]. According to a study conducted by Dekker et al. [10], a low dose of propofol (1 mg/kg) does not induce complications significantly compared to a high dose of propofol (3 to $6 \mathrm{mg} / \mathrm{kg}$ ). Based on these evidences, we decided to inject a low dose of propofol (bolus $1 \mathrm{mg} / \mathrm{kg}$, continuous infusion $100 \mathrm{mcg} / \mathrm{kg} /$ min). We were able to complete the MRI scan without adverse events.

Careful pre-sedation assessment, continuous monitoring, which sedatives to be used, and by whom the sedation is provided are critical factors in pediatric sedation. We successfully sedated a preterm neonate with frequent apnea by performing intubation without a muscle relaxant and maintaining her self-respiration during an MRI scan. We recommend this technique for the seda- tion of preterm infants who have a high risk of respiratory complications.

\section{CONFLICT OF INTEREST}

No potential conflict of interest relevant to this article was reported.

\section{ACKNOWLEDGMENTS}

We would like to thank nurses in the post-anesthesia care unit for their help.

\section{REFERENCES}

1. Tusor N, Arichi T, Counsell SJ, Edwards AD. Brain development in preterm infants assessed using advanced MRI techniques. Clin Perinatol 2014;41:25-45.

2. Arlachov Y, Ganatra RH. Sedation/anaesthesia in paediatric radiology. $\mathrm{Br}$ J Radiol 2012;85:e1018-31.

3. Yamamura K, Takatsu Y, Miyati T, Inatomi T. Brain magnetic resonance imaging using a customized vacuum shape-keeping immobilizer without sedation in preterm infants. Magn Reson Imaging 2018;54:171-5.

4. Guideline for monitoring and management of pediatric patients before, during, and after sedation for diagnostic and therapeutic procedures: update 2016. Pediatr Dent 2016;38:77-106.

5. Machata AM, Willschke H, Kabon B, Kettner SC, Marhofer P. Propofolbased sedation regimen for infants and children undergoing ambulatory magnetic resonance imaging. Br J Anaesth 2008;101:239-43.

6. Dalal PG, Murray D, Cox T, McAllister J, Snider R. Sedation and anesthesia protocols used for magnetic resonance imaging studies in infants: provider and pharmacologic considerations. Anesth Analg 2006;103:863-8.

7. Arthurs OJ, Sury M. Anaesthesia or sedation for paediatric MRI: advantages and disadvantages. Curr Opin Anaesthesiol 2013;26:489-94.

8. Gozal D, Mason KP. Pediatric sedation: a global challenge. Int J Pediatr 2010;2010:701257.

9. Allegaert K, Peeters MY, Verbesselt R, Tibboel D, Naulaers G, de Hoon $\mathrm{JN}$, et al. Inter-individual variability in propofol pharmacokinetics in preterm and term neonates. Br J Anaesth 2007;99:864-70.

10. Dekker J, Lopriore E, Rijken M, Rijntjes-Jacobs E, Smits-Wintjens V, Te Pas A. Sedation during minimal invasive surfactant therapy in preterm infants. Neonatology 2016;109:308-13. 